\title{
REMARKS ON FORCED EQUATIONS OF THE DOUBLE PENDULUM TYPE
}

\author{
GABRIELLA TARANTELLO
}

\begin{abstract}
Motivated by the double pendulum equation we consider Lagrangian systems with potential $V=V(t, q)$ periodic in each of the variables $t$, $q=\left(q_{1}, \ldots, q_{N}\right)$. We study periodic solutions for the corresponding equation of motion subject to a periodic force $f=f(t)$. If $f$ has mean value zero, the corresponding variational problem admits a $\mathbf{Z}^{N}$ symmetry which yields $N+1$ distinct periodic solutions (see [9]). Here we consider the case where the average of $f$, though bounded, is no longer required to be zero. We show how this situation becomes more delicate, and in general it is only possible to claim no more than two periodic solutions.
\end{abstract}

\section{INTRODUCTION}

Let $q, \xi \in \mathbf{R}^{N}$ and $t \in \mathbf{R}$. Given the potential $V=V(t, q)$, consider the time-dependent Lagrangian

$$
\mathscr{L}(q, \xi, t)=\frac{1}{2} A(t, q) \xi \cdot \xi-V(t, q)
$$

(Here $\cdot$ stands for the usual scalar product in $\mathbf{R}^{N}$.) where $A=A(t, q)$ is a symmetric, positive definite $N \times N$ matrix.

The equation of motion for the corresponding mechanical system subject to the forcing $f=f(t) \in \mathbf{R}^{N}$ is given by

$$
\frac{d}{d t} \frac{\partial \mathscr{L}}{\partial \xi}(q, \dot{q}, t)-\frac{\partial \mathscr{L}}{\partial q}(q, \dot{q}, t)=f(t) .
$$

Assuming $A=A(t, q), V=V(t, q)$, and $f=f(t)$ time-periodic with same period $T$, a natural question to ask is whether or not $(0.2)$ admits $T$-periodic solutions. Obviously the answer depends upon the nature of the potential $V$.

Motivated by the double pendulum equation we shall consider periodic potentials. More precisely, given $T_{k}>0, k=1, \ldots, N$, assume

$$
\begin{aligned}
& V=V(t, q) \in C^{1} \text { and } V\left(t+k T, q+\left(k_{1} T_{1}, \ldots, k_{N} T_{N}\right)\right)= \\
& V(t, q) \quad \forall(t, q) \in \mathbf{R} \times \mathbf{R}^{N} \text { and } k, k_{s} \in \mathbf{Z}, s=1, \ldots, N ; \\
& A=A(t, q) \in C^{1} \text { is symmetric positive definite } N \times N \text { matrix } \\
& \text { and } A\left(t+k T, q+\left(k_{1} T_{1}, \ldots, k_{N} T_{N}\right)\right)=A(t, q) \quad \forall(t, q) \in \mathbf{R} \times \\
& \mathbf{R}^{N}, k, k_{s} \in \mathbf{Z}, s=1, \ldots, N .
\end{aligned}
$$

Received by the editors July 13, 1989.

1980 Mathematics Subject Classification (1985 Revision). Primary 34C25, 58E05, 58F05, 58F22. 
This situation has attracted the attention of several authors (e.g., $[2,3,4,8])$ and multiple forced oscillations have been obtained for $(0.2)$ provided the forcing term $f=f(t)$ has mean value zero (i.e., $\left.\int_{0}^{T} f(t) d t=0\right)$. Here we investigate the problem without this restriction on the mean value of $f$. However, our physical intuition suggests that $\left|\frac{1}{T} \int_{0}^{T} f\right|$ cannot be too large in order for $(0.2)$ to admit periodic solutions.

This can be seen rigorously if, for example, we consider the Lagrangian

$$
\begin{aligned}
\mathscr{L}\left(\theta, \phi, \xi_{1}, \xi_{2}\right)= & \frac{1}{2}\left(\left(m_{1}+m_{2}\right) l_{1}^{2} \xi_{1}^{2}+2 m_{2} l_{1} l_{2} \cos (\theta-\phi) \xi_{1} \xi_{2}+m_{2} l_{2}^{2} \xi_{2}^{2}\right) \\
& +g\left(m_{1}+m_{2}\right) l_{1} \cos \theta+g m_{2} l_{2} \cos \phi \\
& (g=\text { constant of gravitation })
\end{aligned}
$$

corresponding to a coplanar double pendulum with masses $m_{i}$ and length $l_{i}$, $i=1,2$. Indeed, if $f(t)=\left(f_{1}(t), f_{2}(t)\right)$, then in this case $(0.2)$ reduces to

$$
\begin{aligned}
& \frac{d}{d t}\left(\left(m_{1}+m_{2}\right) l_{1}^{2} \dot{\theta}+m_{2} l_{1} l_{2} \cos (\phi-\theta) \dot{\phi}\right) \\
& \quad-m_{2} l_{1} l_{2} \sin (\phi-\theta) \dot{\theta} \dot{\phi}+g\left(m_{1}+m_{2}\right) l_{1} \sin \theta=f_{1}, \\
& \frac{d}{d t}\left(m_{2} l_{1} l_{2} \cos (\phi-\theta) \dot{\theta}+m_{2} l_{2}^{2} \dot{\phi}\right) \\
& \quad+m_{2} l_{1} l_{2} \sin (\phi-\theta) \dot{\theta} \dot{\phi}+g m_{2} l_{2} \sin \phi=f_{2} .
\end{aligned}
$$

So, summing up the equations in $(0.3)$ and integrating in $[0, T]$ we see that for $(0.3)$ to admit $T$-periodic solutions, it is necessary that

$$
\frac{1}{T} \int_{0}^{T} f_{1}+\frac{1}{T} \int_{0}^{T} f_{2}=g\left(\left(m_{1}+m_{2}\right) l_{1} \sin \tau_{1}+m_{2} l_{2} \sin \tau_{2}\right)
$$

for suitable $\tau_{1}, \tau_{2} \in[0,2 \pi)$. This condition, however, is not sufficient in general. In fact, for example, if we take $\frac{1}{T} \int_{0}^{T} f_{1}=g\left(m_{1}+m_{2}\right) l_{1}$ and $\frac{1}{T} \int_{0}^{1} f_{2}=$ $g m_{2} l_{2}$ then $(0.3)$ admits a $T$-periodic solution only if $f_{1}(t)=g\left(m_{1}+m_{2}\right) l_{1}$ and $f_{2}(t)=g m_{2} l_{2} \quad \forall t$. Thus the search of $T$-periodic solutions for $(0.2)$ becomes a delicate problem, especially when $\int_{0}^{T} f \neq 0$. To the author's knowledge the only known results in this direction are those obtained in [9] specifically for the $N$-pendulum equations.

Here we treat the case where only one of the $f$ components, say the first one, has mean values zero. So we shall write $f(t)=\left(f_{1}(t)+c, f_{2}(t), \ldots, f_{N}(t)\right)$ with $\int_{0}^{T} f_{k}=0, k=1, \ldots, N$ and $c \in \mathbf{R}$.

Notice that under the given assumptions the problem admits a $\mathbf{Z}^{N}$ symmetry, in the sense that if $q=q(t)$ is a $T$-periodic solution for $(0.2)$ so it is $q(t)+$ $\left(k_{1} T_{1}, \ldots, k_{N} T_{N}\right) \quad \forall k_{s} \in \mathbf{Z}, s=1, \ldots, N$. This motivates the following

Definition. $q_{1}=q_{1}(t)$ and $q_{2}=q_{2}(t)$ are called distinct if

$$
q_{1}(t)-q_{2}(t) \notin\left\{\left(k_{1} T_{1}, \ldots, k_{n} T_{n}\right) \forall k_{s} \in \mathbf{Z}, s=1, \ldots, N\right\}
$$

for all $t \in[0, T]$. 
To clarify the content of our main result (Theorem 1 below) we start with some preliminary observations. First of all we have a variational principle associated with $(0.2)$. Hence $T$-periodic solutions of $(0.2)$ are critical points of a suitable functional $I_{c}$ defined in the Hilbert space

$$
H=\left\{q=\left(q_{1}, \ldots, q_{N}\right): q_{k} \in H^{1}([0, T]), q_{k}(0)=q_{k}(T), k=1, \ldots, N\right\}
$$

with $I_{c}(q)=I_{0}(q)+c \int_{0}^{T} q_{1}$ for all $q=\left(q_{1}, \ldots, q_{N}\right) \in H$.

Now, if $c=0$ (i.e., $\int_{0}^{T} f=0$ ) then $I_{0}$ is in fact well defined (and bounded below) in the Hilbert manifold $M=T^{N} \times H_{\#}$ where $T^{N}$ is the $N$-dimensional torus and $H_{\#}=\left\{q \in H: \int_{0}^{T} q=0\right\}$. So by the Ljusternik-Schnirelman theory one concludes that $I_{0}$ admits at least $N+1$ ( = cup length $T^{N}+1$ ) distinct critical points (see $[7,8]$ and $[3,4]$ for sharper results concerning the $N$-pendulum equation). This is no longer available when $c \neq 0$, and in fact the behavior of $I_{c}$, as far as critical points are concerned, can be completely different from that of $I_{0}$ even for very small $c$. This is illustrated by the following finitedimensional examples where, in analogy, we investigate the critical points for functions of the type $G_{c}\left(x_{1}, \ldots, x_{N}\right)=g\left(x_{1}, \ldots, x_{N}\right)+c x_{1}$ with $g$ periodic in each variable.

Example 1. Take $\psi=\psi(t)$ to be a smooth 1-periodic function. Given $T_{k}>0$, $k=1, \ldots, N$, set

$$
g\left(x_{1}, \ldots, x_{N}\right)=\psi\left(\sum_{k=1}^{N} T_{k}^{-1} x_{k}\right) .
$$

So $g$ is $T_{k}$ periodic in $x_{k}$. It is easy to see that while $g$ admits infinitely many critical points, $G_{c}\left(x_{1}, \ldots, x_{N}\right)=g\left(x_{1}, \ldots, x_{N}\right)+c x_{1}$ has no critical points for all $c \neq 0$.

Example 2. Take

$$
\begin{aligned}
g\left(x_{1}, \ldots, x_{N}\right)= & \sin x_{1}\left(\sin x_{2}-1\right) \cdot\left(\sin x_{3}-1\right) \\
& \cdots\left(\sin x_{N}-1\right)+2^{N-1} \sum_{k=2}^{N} \sin x_{k}
\end{aligned}
$$

so $g$ is $2 \pi$-periodic in $x_{k}, k=1, \ldots, N$.

Easy computations show that $g$ has infinitely many critical points in $[0,2 \pi)$. However $G_{c}\left(x_{1}, \ldots, x_{N}\right)=g\left(x_{1}, \ldots, x_{N}\right)+c x_{N}$ admits exactly $t w o$ (nondegenerate) critical points in $[0,2 \pi)$ if $|c|<2^{N-1}$, exactly one if $|c|=2^{N-1}$, and none if $|c|>2^{N-1}$.

These two examples justify the following.

Theorem 1. Let $V=V(t, q)$ and $A=A(t, q)$ satisfy $(V)$ and $(A)$, respectively, and $f_{k}=f_{k}(t)$ be a $T$-periodic continuous function with $\int_{0}^{T} f_{k}=0$, 
$k=1, \ldots, N$. There exist constants $d \leq 0 \leq D$ (depending on $V, A$, and $f_{k}$ ) with the following property:

(i) if $d=D$ then for all $\xi \in \mathbf{R}$ problem $(0.2)$ with $f(t)=\left(f_{1}(t), \ldots, f_{N}(t)\right)$ admits a T-periodic solution $q_{\xi}(t)=\left(q_{1, \xi}(t), \ldots, q_{N, \xi}(t)\right)$ with $\frac{1}{T} \int_{0}^{T} q_{1, \xi}=\xi$

(ii) if $d<D$ then problem (0.2) with $f(t)=\left(f_{1}(t)+c, \ldots, f_{N}(t)\right)$ admits at least two distinct solutions if $c \in(d, D)$, and at least one solution if $c=d$ or $c=D$.

Remark. Restrictions on $c$ were expected by the previous observations. Furthermore, by virtue of Examples 1 and 2 we know that in general the statement cannot be improved. However, we shall see how, in certain cases, it is possible to rule out the possibility $d=0=D$ and give estimates on $d$ and $D$. This will be the content of Theorem 2 below. Here we only state its particular implication to the double pendulum equation.

Corollary. Let $\left.e(t)=\left(e_{1}(t)\right), e_{2}(t)\right)$ be a continuous $T$-periodic function with $\int_{0}^{T} e_{k}=0, k=1,2$, and $c \in \mathbf{R}$. Assume

(a) $\left(m_{1}+m_{2}\right) l_{1}-m_{2} l_{2}:=\gamma>0$ and

(b) $\left(\left(m_{1}+m_{2}\right) l_{1} / 2 \lambda_{0} \pi^{2}\right)\left(\|e\|_{2}+\sqrt{T}\left(m_{1}+m_{2}\right) l_{1}\right) T^{3 / 2}:=\sigma_{T}<\gamma$ with

$$
\lambda_{0}=\frac{1}{2}\left(m_{2} l_{2}^{2}+\left(m_{1}+m_{2}\right) l_{1}^{2}-\sqrt{\left(m_{2} l_{2}^{2}-\left(m_{1}+m_{2}\right) l_{1}^{2}\right)^{2}+4 m_{2}^{2} l_{1}^{2} l_{2}^{2}}\right) .
$$

There exist constants $d<0<D$ (depending on $e_{k}, m_{k}, l_{k}, k=1,2$, and $T$ ) such that equation (0.3) with $f_{1}(t)=e_{1}(t)+c$ and $f_{2}(t)=e_{2}(t)$ admits at least two distinct solutions if $c \in(d, D)$ and at least one solution if $c=d$ or $c=D$. Furthermore, $d \leq-\gamma+\sigma_{T}<0<\gamma-\sigma_{T} \leq D$.

Similar conditions were introduced in [4] to obtain multiple forced oscillations for the double pendulum equation in case $c=0$. See [3], also, for extensions to the $N$-pendulum case. Furthermore, it has been observed in [9] that pathologies of the kind portrayed by Examples 1 and 2 cannot occur for the $N$-pendulum equation, where much stronger multiplicity results hold provided $m_{i}, l_{1}, e_{i}(t)$, and $T$ satisfy suitable conditions. For the simple pendulum equation, a result of the type of Theorem 1 was obtained by Mawhin-Willem [7] (see also [11]).

The proof of Theorem 1 and applications. Set $f(t)=\left(f_{1}(t)+c, \ldots, f_{N}(t)\right)$ with $f_{k} \in L^{2}[0, T], \int_{0}^{T} f_{k}=0, k=1, \ldots, N$, and $c \in \mathbf{R}$. We seek solutions for the following problem

$$
\left\{\begin{array}{l}
\frac{d}{d t} \frac{\partial \mathscr{L}}{\partial \xi_{1}}(q, \dot{q}, t)-\frac{\partial \mathscr{L}}{\partial q_{1}}(q, \dot{q}, t)=f_{1}(t)+c, \\
\frac{d}{d t} \frac{\partial \mathscr{L}}{\partial \xi_{k}}(q, \dot{q}, t)-\frac{\partial \mathscr{L}}{\partial q_{k}}(q, \dot{q}, t)=f_{k}(t), \quad k=2, \ldots, N, \\
q(0)=q(T), \quad \dot{q}(0)=\dot{q}(T) .
\end{array}\right.
$$


As it is well known from classical mechanics, (weak) solutions of $(1)_{c}$ are the critical points of the functional

$$
I_{c}(q)=\frac{1}{2} \int_{0}^{T} A(t, q) \dot{q} \cdot \dot{q}-\int_{0}^{T} V(t, q)+\sum_{k=1}^{N} \int_{0}^{T} f_{k} q_{k}+c \int_{0}^{T} q_{1}
$$

defined in the Hilbert space

$$
H=\left\{q=\left(q_{1}, \ldots, q_{N}\right): q_{k} \in H^{1}([0, T]), q_{k}(0)=q_{k}(T), k=1, \ldots, N\right\}
$$

equipped with the standard scalar product

$$
(q, Q)=\int_{0}^{T} \dot{q} \cdot \dot{Q}+\int_{0}^{T} q \cdot Q, \quad q, Q \in H,
$$

and norm $\|q\|=(q, q)^{1 / 2}$. Notice that for $c \neq 0, I_{c}$ is unbounded in $H$ and multivalued in $M=T^{N} \times H_{\#}$.

For $q=\left(q_{1}, \ldots, q_{N}\right) \in H$, set

$$
\|q\|_{2}=\left(\sum_{k=1}^{N}\left\|q_{k}\right\|_{L^{2}}^{2}\right)^{1 / 2} \text { and }\|q\|_{\infty}=\sum_{k=1}^{N}\left\|q_{k}\right\|_{L^{\infty}} .
$$

Moreover, since $A=A(t, q)$ is positive definite, let $\lambda_{0}>0$ satisfy $A(t, q) \xi \cdot \xi$ $\geq \lambda_{0}|\xi|^{2} \quad \forall t \in \mathbf{R}, q, \xi \in \mathbf{R}^{N}$. For every $c \in \mathbf{R}$, we have that $I_{c}$ satisfies a Palais-Smale type condition. More precisely,

Lemma 1.1. Any sequence $q_{n} \in H$ satisfying.

(i) $\left|\frac{1}{T} \int_{0}^{T} q_{n}\right| \leq C_{1}$ for all $n \in \mathbf{N}$ ( $C_{1}$ positive constant),

(ii) $I_{c}\left(q_{n}\right) \leq C_{2}$ for all $n \in \mathbf{N}$ ( $C_{2}$ positive constant),

(iii) $\left(I_{c}^{\prime}\left(q_{n}\right)-I_{c}^{\prime}\left(q_{m}\right)\right)\left(q_{n}-q_{m}\right) \rightarrow 0$ as $n, m \rightarrow+\infty$, admits a convergent subsequence in $H$.

Proof. Since the potential $V$ is bounded, set $|V(t, q)| \leq V_{0} \quad \forall(t, q) \in \mathbf{R} \times \mathbf{R}^{N}$ for a suitable constant $V_{0}>0$. Furthermore, let $f_{0}(t)=\left(f_{1}(t), \ldots, f_{N}(t)\right)$ and $q_{n}=q_{n}^{0}+\xi_{n}$ with $\xi_{n}=\left(\xi_{1, n}, \ldots, \xi_{N, n}\right) \in \mathbf{R}^{N}$ and $\int_{0}^{T} q_{n}^{0}=0$. We have

$$
\begin{aligned}
C_{2} \geq & I_{c}\left(q_{n}\right)=\frac{1}{2} \int_{0}^{T} A\left(t, q_{n}\right) \dot{q}_{n} \cdot \dot{q}_{n}-\int_{0}^{T} V\left(t, q_{n}\right) \\
& +\int_{0}^{T} f_{0} \cdot q_{n}^{0}+c \int_{0}^{T} q_{1} \\
\geq & \frac{\lambda_{0}}{2}\left\|\dot{q}_{n}\right\|_{2}^{2}-\left\|f_{0}\right\|_{2}\left\|q_{n}^{0}\right\|_{2}-\left(V_{0}+|c| C_{1}\right) T \\
\geq & \frac{\lambda_{0}}{2}\left\|\dot{q}_{n}\right\|^{2}-\frac{T\left\|f_{0}\right\|_{2}}{2 \pi}\left\|\dot{q}_{n}\right\|_{2}-\left(V_{0}+|c| C_{1}\right) T .
\end{aligned}
$$

This yields $\left\|\dot{q}_{n}\right\|_{2} \leq c_{1} \forall n \in \mathbf{N}$ for some suitable constant $c_{1}>0$. This fact, together with (i) implies that for a subsequence of $q_{n}$ (which we still call $q_{n}$ ) and $q \in H$ we have $\left\|q_{n}-q\right\|_{\infty} \rightarrow 0$ as $n \rightarrow+\infty$. Set

$$
A_{n}(t)=\left(\frac{\partial A}{\partial q_{1}}\left(t, q_{n}\right) \dot{q}_{n} \cdot \dot{q}_{n}, \ldots, \frac{\partial A}{\partial q_{N}}\left(t, q_{n}\right) \dot{q}_{n} \cdot \dot{q}_{n}\right) \in \mathbf{R}^{N} .
$$


Obviously,

$$
\sum_{k=1}^{N}\left\|\frac{\partial A}{\partial q_{k}}\left(t, q_{n}\right) \dot{q}_{n} \cdot \dot{q}_{n}\right\|_{L^{1}} \leq c_{2} \quad \forall n \in \mathbf{N}
$$

for $c_{2}>0$ suitable constant. Furthermore, by the Lipschitz continuity of $A=A(t, q)$ in $q$ (uniformly in $t$ ) we have

$$
\begin{aligned}
\left(I_{c}^{\prime}\left(q_{n}\right)\right. & \left.-I_{c}^{\prime}\left(q_{m}\right)\right)\left(q_{n}-q_{m}\right)=\int_{0}^{T} A\left(t, q_{n}\right)\left(\dot{q}_{n}-\dot{q}_{m}\right) \cdot\left(\dot{q}_{n}-\dot{q}_{m}\right) \\
& +\int_{0}^{T}\left(A\left(t, q_{n}\right)-A\left(t, q_{m}\right)\right) \dot{q}_{m} \cdot\left(\dot{q}_{n}-\dot{q}_{m}\right) \\
& +\frac{1}{2} \int_{0}^{T}\left(A_{n}(t)-A_{m}(t)\right) \cdot\left(q_{n}-q_{m}\right) \\
& +\int_{0}^{T}\left(\frac{\partial V}{\partial q}\left(t, q_{n}\right)-\frac{\partial V}{\partial q}\left(t, q_{m}\right)\right) \cdot\left(q_{n}-q_{m}\right) \\
\geq & \lambda_{0}\left\|\dot{q}_{n}-\dot{q}_{m}\right\|_{2}^{2}-c_{3}\left\|q_{n}-q_{m}\right\|_{\infty} \quad\left(c_{3} \text { positive constant }\right) .
\end{aligned}
$$

Consequently, $\left\|\dot{q}_{n}-\dot{q}_{m}\right\|_{2} \rightarrow 0$ as $n, m \rightarrow+\infty$, and therefore $q_{n} \rightarrow q$ in $H$.

Given $\xi \in \mathbf{R}$ set $\Lambda_{\xi}=\left\{q=\left(q_{1}, \ldots, q_{N}\right) \in H: \frac{1}{T} \int_{0}^{T} q_{1}=\xi\right\}$. For every $c \in \mathbf{R}, I_{c}$ is bounded from below in $\Lambda_{\xi}$. Furthermore,

Lemma 1.2. For every $\xi \in \mathbf{R}$, there exists $q_{\xi} \in \Lambda_{\xi}$ such that

$$
I_{0}\left(q_{\xi}\right)=\inf _{\Lambda_{\xi}} I_{0} .
$$

Moreover, $\operatorname{Inf}_{\Lambda_{\xi}} I_{c}=I_{0}\left(q_{\xi}\right)+c T \xi=I_{c}\left(q_{\xi}\right)$.

Proof. Set $m_{\xi}=\inf _{\Lambda_{\xi}} I_{0}$ and let $q_{n}=\left(q_{1, n}, \ldots, q_{N, n}\right) \in \Lambda_{\xi}$ such that

$$
\lim _{n \rightarrow+\infty} I_{0}\left(q_{n}\right)=m_{\xi} \text {. }
$$

Since $I_{0}(q)=I_{0}\left(q+\left(k_{1} T_{1}, \ldots, k_{n} T_{n}\right)\right)$ for all $k_{s} \in \mathbf{Z}, s=1, \ldots, N$, we can always assume that $\frac{1}{T} \int_{0}^{T} q_{k, n} \in\left[0, T_{k}\right], k=2, \ldots, N$. So

$$
\left|\frac{1}{T} \int_{0}^{T} q_{n}\right| \leq|\xi|+\sum_{k=2}^{N} T_{k} .
$$

Furthermore from (1.2) it follows that $\left\|\dot{q}_{n}\right\|_{L^{2}} \leq C \quad \forall n \in \mathbf{N}$, for a suitable positive constant $C$. Hence for a subsequence $\left\{q_{n_{k}}\right\}$ of $\left\{q_{k}\right\}$ and $q_{0} \in H$ we have $\dot{q}_{n_{k}} \rightarrow \dot{q}_{0}$ weakly in $L^{2}$ and $q_{n_{k}} \rightarrow q_{0}$ strongly in $L^{2}$.

Consequently $q_{0} \in \Lambda_{\xi}$. The lower semicontinuity of $I_{0}$ yields the conclusion.

Our next goal is to obtain a priori estimates on $\dot{q}_{\xi}$ independent of $\xi$. To this purpose let $L$ be the Lipschitz constant of $V$, i.e.,

$$
|V(t, q)-V(t, Q)| \leq L|q-Q|
$$

for all $t \in \mathbf{R}, q, Q \in \mathbf{R}^{N}$. Set $f_{0}(t)=\left(f_{1}(t), \ldots, f_{N}(t)\right)$. We have 
Lemma 1.3. If $q_{\xi} \in \Lambda_{\xi}$ satisfies $(1.1)_{\xi}$ then

$$
\left\|\dot{q}_{\xi}\right\|_{2} \leq \frac{T}{\pi \lambda_{0}}\left(\left\|f_{0}\right\|_{2}+\sqrt{T} L\right) .
$$

Proof. Set $q_{\xi}=q_{\xi}^{0}+\left(\xi, \sigma_{2}, \ldots, \sigma_{N}\right)$ with $\int_{0}^{T} q_{\xi}^{0}=0$. Since $T V\left(\xi, \sigma_{2}, \ldots, \sigma_{N}\right)$ $=I_{0}\left(\xi, \sigma_{2}, \ldots, \sigma_{N}\right) \geq I_{0}\left(q_{\xi}\right)$, we have

$$
\begin{aligned}
0 \geq & \frac{1}{2} \int_{0}^{T} A\left(t, q_{\xi}\right) \dot{q}_{\xi} \cdot \dot{q}_{\xi} \\
& \quad-\int_{0}^{T}\left(V\left(q_{\xi}^{0}+\left(\xi, \sigma_{2}, \ldots, \sigma_{N}\right)\right)-V\left(\xi, \sigma_{2}, \ldots, \sigma_{N}\right)\right)+\int_{0}^{T} f_{0} \cdot q_{\xi}^{0} \\
\geq & \frac{\lambda_{0}}{2}\left\|\dot{q}_{\xi}\right\|_{2}^{2}-L \sqrt{T}\left\|q_{\xi}^{0}\right\|_{2}-\left\|f_{0}\right\|_{2}\left\|q_{\xi}^{0}\right\|_{2} .
\end{aligned}
$$

Thus

$$
0 \geq\left\|\dot{q}_{\xi}\right\|_{2}\left(\frac{\lambda_{0}}{2}\left\|\dot{q}_{\xi}\right\|_{2}-\frac{T}{2 \pi}\left(\left\|f_{0}\right\|_{2}+L \sqrt{T}\right)\right) .
$$

For $\xi \in \mathbf{R}$ set $\Gamma_{\xi}=\left\{q \in \Lambda_{\xi}: I_{0}(q)=\inf _{\Lambda_{\xi}} I_{0}\right\}$. By Lemma 1.2 we know that $\Gamma_{\xi} \neq \varnothing$ for all $\xi \in \mathbf{R}$.

Lemma 1.4. For every $c \in \mathbf{R}$, there exists $L_{c}>0$ such that

$$
\left|I_{c}\left(q_{\xi_{1}}+\sigma_{1}\right)-I_{c}\left(q_{\xi_{2}}+\sigma_{2}\right)\right| \leq L_{c}\left(\left\|q_{\xi_{1}}-q_{\xi_{2}}\right\|+\left|\sigma_{1}-\sigma_{2}\right|\right)
$$

for all $\sigma_{i} \in \mathbf{R}^{N}, \xi_{i} \in \mathbf{R}, q_{\xi_{i}} \in \Gamma_{\xi_{i}}, i=1,2$.

Proof. Let $L^{\prime}$ be the (uniform) Lipshitz constant corresponding to $A(t, q)$ and let $A_{0}$ satisfy $|A(t, q) \xi \cdot \eta| \leq A_{0}|\xi||\eta|$ for all $t \in \mathbf{R}, q, \xi, \eta \in \mathbf{R}^{N}$. Set $\hat{q}_{\xi_{i}}=q_{\xi_{i}}+\sigma_{i}, i=1,2$. We have

$$
\begin{aligned}
\mid I_{c}\left(\hat{q}_{\xi_{1}}\right) & -I_{c}\left(\hat{q}_{\xi_{2}}\right) \mid \\
\leq & \frac{1}{2} \int_{0}^{T}\left|A\left(t, \hat{q}_{\xi_{1}}\right) \dot{q}_{\xi_{1}} \cdot \dot{q}_{\xi_{1}}-A\left(t, \hat{q}_{\xi_{2}}\right) \dot{q}_{\xi_{2}} \cdot \dot{q}_{\xi_{2}}\right| \\
& +\int_{0}^{T}\left|V\left(t, \hat{q}_{\xi_{1}}\right)-V\left(t, \hat{q}_{\xi_{2}}\right)\right|+\int_{0}^{T}\left|f_{0} \cdot\left(q_{\xi_{1}}^{0}-q_{\xi_{2}}^{0}\right)\right| \\
& +T|c|\left(\left|\xi_{1}-\xi_{2}\right|+\left|\sigma_{1}-\sigma_{2}\right|\right) \\
\leq & \frac{1}{2} \int_{0}^{T}\left|\left(A\left(t, \hat{q}_{\xi_{1}}\right)-A\left(t, \hat{q}_{\xi_{2}}\right)\right) \dot{q}_{\xi_{1}} \cdot \dot{q}_{\xi_{1}}\right|+\frac{1}{2} \int_{0}^{T}\left|A\left(t, \hat{q}_{\xi_{2}}\right) \dot{q}_{\xi_{1}} \cdot\left(\dot{q}_{\xi_{1}}-\dot{q}_{\xi_{2}}\right)\right| \\
& +\frac{1}{2} \int_{0}^{T}\left|A\left(t, \hat{q}_{\xi_{2}}\right) \dot{q}_{\xi_{2}} \cdot\left(\dot{q}_{\xi_{1}}-\dot{q}_{\xi_{2}}\right)\right|+L \sqrt{T}\left\|\hat{q}_{\xi_{1}}-\hat{q}_{\xi_{2}}\right\|_{2} \\
& +\frac{T}{2 \pi}\left\|f_{0}\right\|_{2}\left\|\dot{q}_{\xi_{1}}-\dot{q}_{\xi_{2}}\right\|_{2}+|c| T\left(\left|\xi_{1}-\xi_{2}\right|+\left|\sigma_{1}-\sigma_{2}\right|\right) \\
\leq & \frac{L^{\prime}}{2}\left\|\hat{q}_{\xi_{1}}-\hat{q}_{\xi_{2}}\right\|_{\infty}\left\|\dot{q}_{\xi_{1}}\right\|_{2}^{2}+\frac{A_{0}}{2}\left\|\dot{q}_{\xi_{1}}\right\|_{2}\left\|\dot{q}_{\xi_{1}}-\dot{q}_{\xi_{2}}\right\|_{2}+\frac{A_{0}}{2}\left\|\dot{q}_{\xi_{2}}\right\|_{2}\left\|\dot{q}_{\xi_{1}}-\dot{q}_{\xi_{2}}\right\|_{2} \\
& +\sqrt{T}(L+|c|)\left\|q_{\xi_{1}}-q_{\xi_{2}}\right\|_{2}+\frac{T}{2 \pi}\left\|f_{0}\right\|_{2}\left\|\dot{q}_{\xi_{1}}-\dot{q}_{\xi_{2}}\right\|_{2}+T(|c|+L)\left|\sigma_{1}-\sigma_{2}\right| .
\end{aligned}
$$


Hence by the estimate of Lemma 1.3 we conclude that

$$
\begin{aligned}
\left|I_{c}\left(q_{\xi_{1}}\right)-I_{c}\left(q_{\xi_{2}}\right)\right| \leq & \frac{L^{\prime}}{2}\left(\frac{T}{\pi \lambda_{0}}\left(\left\|f_{0}\right\|_{2}+\sqrt{T} L\right)\right)^{2}\left(\left\|q_{\xi_{1}}-q_{\xi_{2}}\right\|_{\infty}+\left|\sigma_{1}-\sigma_{2}\right|\right) \\
& +\left(A_{0} \frac{T}{\pi \lambda_{0}}\left(\left\|f_{0}\right\|_{2}+\sqrt{T} L\right)+\frac{T}{2 \pi}\left\|f_{0}\right\|_{2}\right)\left\|\dot{q}_{\xi_{1}}-\dot{q}_{\xi_{2}}\right\|_{2} \\
& +\sqrt{T}(L+|c|)\left(\left\|q_{\xi_{1}}-q_{\xi_{2}}\right\|_{2}+\sqrt{T}\left|\sigma_{1}-\sigma_{2}\right|\right) \\
\leq & L_{c}\left(\left\|q_{\xi_{1}}-q_{\xi_{2}}\right\|+\left|\sigma_{1}-\sigma_{2}\right|\right)
\end{aligned}
$$

for some suitable constant $L_{c}>0$ independent of $\xi_{i}$ and $\sigma_{i}, i=1,2$.

Proof of Theorem 1. Given $q \in H$, define

$$
\psi(q)=-\frac{1}{T}\left(\frac{1}{2} \int_{0}^{T} \frac{\partial A}{\partial q_{1}}(t, q) \dot{q} \cdot \dot{q}-\int_{0}^{T} \frac{\partial V}{\partial q_{1}}(t, q)\right) .
$$

Set $d=\inf _{\xi \in \mathbf{R}} \inf _{q \in \Gamma_{\xi}} \psi(q)$ and $D=\sup _{\xi \in \mathbf{R}} \sup _{q \in \Gamma_{\xi}} \psi(q)$. By Lemma 1.3 we know that $-\infty<d \leq D<+\infty$. Furthermore, $I_{0}$ is bounded from below in $H$, and by Lemma 1.1 it follows that its minimum is achieved at some point $q_{0} \in H$. In particular $\psi\left(q_{0}\right)=0$. So $d \leq \psi\left(q_{0}\right)=0 \leq D$. Notice that $\forall \xi \in \mathbf{R}$ and $q_{\xi} \in \Gamma_{\xi}$ we have $I_{0}^{\prime}\left(q_{\xi}\right)=-T\left(\psi\left(q_{\xi}\right), 0, \ldots, 0\right) \in \mathbf{R}^{N}$. Therefore if $d=D=0$, then for every $\xi \in \mathbf{R}, q_{\xi} \in \Gamma_{\xi}(\neq \varnothing)$ would be a solution for (1) $c=0$ and $\frac{1}{T} \int_{0}^{T} q_{1, \xi}=\xi$. Now assume $d<D$, and let $c \in(d, D)$. There exists $\xi_{1}, \xi_{2} \in \mathbf{R}$ such that $\psi\left(q_{\xi_{2}}\right)<c<\psi\left(q_{\xi_{1}}\right)$ for all $q_{\xi_{1}} \in \Gamma_{\xi_{1}}$ and for all $q_{\xi_{2}} \in \Gamma_{\xi_{2}}$. Since $\psi\left(q_{\xi+k T_{1}}\right)=\psi\left(q_{\xi}\right)$ and $q_{\xi} \in \Gamma_{\xi} \Leftrightarrow q_{\xi+k T_{1}} \in \Gamma_{\xi+k T_{1}}$ for all $k \in \mathbf{Z}$, we can always assume $0<\xi_{2}-\xi_{1}<T_{1}$. Set

$$
\Lambda_{\xi_{1}, \xi_{2}}=\left\{q=\left(q_{1}, \ldots, q_{N}\right) \in H: \xi_{1} \leq \frac{1}{T} \int_{0}^{T} q_{1} \leq \xi_{2}\right\} .
$$

Notice that $I_{c}$ is bounded from below in $\Lambda_{\xi_{1}, \xi_{2}}$. We shall obtain our first solution by showing that $\inf _{\Lambda_{\xi_{1}}, \xi_{2}} I_{c}$ is achieved at an interior point of $\Lambda_{\xi_{1}}, \xi_{2}$. Set $m=\inf _{\Lambda_{\xi_{1}, \xi_{2}}} I_{c}$. Let $q_{n} \stackrel{=}{=}\left(q_{1, n}, \ldots, q_{N, n}\right) \in \Lambda_{\xi_{1}, \xi_{2}} \quad n \in \mathbf{N}$, satisfy $\lim _{n \rightarrow+\infty} I_{c}\left(q_{n}\right)=m$. If $\xi_{n}=\frac{1}{T} \int_{0}^{T} q_{1, n} \in\left[\xi_{1}, \xi_{2}\right]$, without loss of generality we can assume $q_{n}=q_{\xi_{n}} \in \Gamma_{\xi_{n}}$ and $\lim _{n \rightarrow+\infty} \xi_{n}=\xi_{0}$ with $\xi_{1} \leq \xi_{0} \leq \xi_{2}$. In addition, given $q_{\xi_{0}} \in \Gamma_{\xi_{0}}^{n}$, by Lemma 1.4 we have

$$
\begin{aligned}
m & =\inf _{\Lambda_{\xi_{1}}, \xi_{2}} I_{c} \leq I_{c}\left(q_{\xi_{0}}\right) \leq I_{c}\left(q_{\xi_{n}}+\left(\xi_{0}-\xi_{n}, 0, \ldots, 0\right)\right) \\
& =I_{c}\left(q_{\xi_{n}}+\left(\xi_{0}-\xi_{n}, 0, \ldots, 0\right)\right)-I_{c}\left(q_{\xi_{n}}\right)+I_{c}\left(q_{\xi_{n}}\right) \\
& \leq L_{c}\left|\xi_{0}-\xi_{n}\right|+I_{c}\left(q_{\xi_{n}}\right) \rightarrow m \text { as } n \rightarrow+\infty .
\end{aligned}
$$

Therefore $I_{c}\left(q_{\xi_{0}}\right)=m$. We are done if we show $\xi_{1}<\xi_{0}<\xi_{2}$. To this purpose 
set $\mathscr{T}_{1}(s)=I_{c}\left(q_{\xi_{1}}+(s, 0, \ldots, 0)\right)$. We have

$$
\begin{aligned}
\mathscr{T}_{1}^{\prime}(0) & =\frac{1}{2} \int_{0}^{T} \frac{\partial A}{\partial q_{1}}\left(t, q_{\xi_{1}}\right) \dot{q}_{\xi_{1}} \cdot \dot{q}_{\xi_{1}}-\int_{0}^{T} \frac{\partial V}{\partial q_{1}}\left(t, q_{\xi_{1}}\right)+c T \\
& =-T\left(\psi\left(q_{\xi_{1}}\right)-c\right)<0 .
\end{aligned}
$$

Similarly, if $\varphi_{2}(s)=I_{c}\left(q_{\xi_{2}}+(s, 0, \ldots, 0)\right)$ then $\varphi_{2}^{\prime}(0)=-T\left(\psi\left(q_{\xi_{2}}\right)-c\right)>0$. So for small $\varepsilon>0, I_{c}\left(q_{\xi_{0}}\right) \leq I_{c}\left(q_{\xi_{1}}+(\varepsilon, 0, \ldots, 0)\right)<I_{c}\left(q_{\xi_{1}}\right)$ and $I_{c}\left(q_{\xi_{0}}\right) \leq$ $I_{c}\left(q_{\xi_{2}}-(\varepsilon, 0, \ldots, 0)\right)<I_{c}\left(q_{\xi_{2}}\right)$. Consequently $\xi_{0} \neq \xi_{1}$ and $\xi_{0} \neq \xi_{2}$, which yields $I_{c}^{\prime}\left(q_{\xi_{0}}\right)=0$. So $q_{\xi_{0}}$ is a solution for $(1)_{c}$. Moreover, $q_{\xi_{0}}$ is a local minimum for $I_{c}$, so we shall obtain a second solution via a mountain-pass theorem (see [1]). To this purpose notice that $I_{c}(q)=I_{c}\left(q+\left(0, k_{2} T_{2}, \ldots, k_{N} T_{N}\right)\right)$ for all $k_{s} \in \mathbf{Z}, s=2, \ldots, N$.

Consider

$$
\begin{aligned}
\Sigma=\{\gamma:[0 ; 1] \rightarrow H \text { continuous }: & \gamma(0)=q_{\xi_{0}}, \\
\gamma(1) & \left.=q_{\xi_{0}}+\left(0, k_{2} T_{2}, \ldots, k_{N} T_{N}\right) ; k_{s} \in \mathbf{Z}\right\}
\end{aligned}
$$

and set $\alpha=\inf _{\gamma \in \Sigma} \sup _{t \in[0,1]} I_{c}(\gamma(t)) \geq I_{c}\left(q_{\xi_{0}}\right)$. Although $I_{c}$ does not satisfy the Palais-Smale condition in the usual sense, Lemma 1.1 is enough to guarantee a "deformation lemma". It can be obtained by adjusting the standard proof of the deformation theorem as shown in [8, Proposition 1.10], with the further simplification that condition $2^{0}$ of Proposition 1.10 is not needed here. Thus a sharper version of the mountain-pass theorem due to D. Guo, J. Sun, and G. Qi (see [5, Proposition 2]) gives that $\alpha$ is a critical value for $I_{c}$, and that $K_{\alpha}:=\left\{q \in H: I_{c}(q)=\alpha, I_{c}^{\prime}(q)=0\right\}$ contains a critical point different from $q_{\xi_{0}}+\left(k_{1} T_{1}, k_{2} T_{2}, \ldots, k_{N} T_{N}\right)$ for all $k_{s} \in \mathbf{Z}, s=1, \ldots, N$. (This generalizes previous results of Hofer [6].) So we are guaranteed a second distinct solution for $(1)_{c}$.

Finally, if $c=d$ or $c=D$, take a sequence $\left\{c_{n}\right\} \subset(d, D)$ such that $c_{n} \rightarrow d$ (or $c_{n} \rightarrow D$ ) as $n \rightarrow+\infty$. By previous arguments, for every $n \in \mathbf{N}$ there exists $q_{n}=\left(q_{1, n}, \ldots, q_{N, n}\right) \in H$ such that

(a) $\frac{1}{T} \int_{0}^{T} q_{k, n} \in\left[0, T_{k}\right]$ for all $n \in \mathbf{N}$ and for all $k=1, \ldots, N$;

(b) $\left\|\dot{q}_{n}\right\|_{2} \leq \frac{T}{\lambda_{0} \pi}\left(\left\|f_{0}\right\|_{2}+\sqrt{T} L\right)$;

(c) $0=I_{c_{n}}^{\prime}\left(q_{n}\right)=I_{d}^{\prime}\left(q_{n}\right)+c_{n}-d$.

Therefore $\left|\left(I_{d}^{\prime}\left(q_{n}\right)-I_{d}^{\prime}\left(q_{m}\right)\right)\left(q_{n}-q_{m}\right)\right|=\left|c_{n}-c_{m}\right|\left\|q_{n}-q_{m}\right\| \rightarrow 0$ as $n, m \rightarrow+\infty$, since (a) and (b) $\Rightarrow\left\|q_{n}-q_{m}\right\| \leq C$ for all $n, m \in \mathbf{N}(C>0$ constant). Hence as for Lemma 1.1 we obtain a subsequence $\left\{q_{n_{k}}\right\}$ of $\left\{q_{n}\right\}$ and $q \in H: q_{n_{k}} \rightarrow q$ in $H$. Obviously $I_{d}^{\prime}(q)=0$. Completely analogous is the case $c_{n} \rightarrow D$.

Our next goal is to find conditions on the matrix $A=A(t, q)$ and $V=$ $V(t, q)$ which exclude the possibility $d=0=D$. To this end let $V=V(t, q)$ 
have the special form

$$
V(t, q)=g\left(q_{1}\right)+V_{1}(t, q)
$$

where

$(V)_{1} \quad g \in C^{1}$ is $T_{1}$ periodic and $V_{1}$ satisfies $(V)$.

Set $g_{m}^{\prime}=\min _{\mathbf{R}} g^{\prime} \leq 0 \leq \max _{\mathbf{R}} g^{\prime}=g_{M}^{\prime}$,

$$
V_{m}=\min _{\mathbf{R}^{N+1}} \sum_{k=1}^{N} \frac{\partial V_{1}}{\partial q_{k}} \leq \max _{\mathbf{R}^{N+1}} \sum_{k=1}^{N} \frac{\partial V_{1}}{\partial q_{k}}=V_{M}
$$

and $\gamma=\min \left\{-g_{m}^{\prime}-V_{M}, g_{M}^{\prime}+V_{m}\right\}$. Furthermore, let $\lambda_{1} \geq 0$ satisfy

$$
\left|\sum_{k=1}^{N} \frac{\partial A}{\partial q_{k}}(t, q) \xi \cdot \xi\right| \leq \lambda_{1}|\xi|^{2}
$$

We have

Theorem 2. Let $A=A(t, q), f_{k}=f_{k}(t), k=1, \ldots, N$, as in Theorem 1 , and $V=V(t, q)$ of the form (1.5) satisfy $(V)_{1}$. Assume $\gamma>0$ and

$$
\mu_{T}:=\frac{T}{\lambda_{0} \pi^{2}}\left(\left\|f_{0}\right\|_{2}+\sqrt{T} L\right)\left(\frac{\lambda_{1}}{\lambda_{0}}\left(\left\|f_{0}\right\|_{2}+\sqrt{T} L\right)+\frac{l}{2} \sqrt{T}\right)<\gamma
$$

where $L$ and $l$ are the Lipshitz constants of $V$ and $g^{\prime}$ respectively. There exists constants $d<0<D$ such that if $c \in(d, D)$ then problem $(1)_{c}$ admits at least two distinct solutions; and if $c=d$ or $c=D$ then $(1)_{c}$ admits at least one solution. Furthermore; $d \leq g_{m}^{\prime}+V_{M}+\mu_{T}<0<g_{M}^{\prime}+V_{m}-\mu_{T}$.

Proof. First of all, notice that for all $\xi \in \mathbf{R}$ and $q_{\xi} \in \Gamma_{\xi}, \int_{0}^{T} \frac{\partial A}{\partial q_{k}}\left(t, q_{\xi}\right) \dot{q}_{\xi} \cdot \dot{q}_{\xi}-$ $\frac{\partial V}{\partial q_{k}}\left(t, q_{\xi}\right)=0$ for all $k=2, \ldots, N$. Let $\xi_{0} \in\left[0, T_{1}\right]$ with $g^{\prime}\left(\xi_{0}\right)=g_{m}^{\prime}$. We have

$$
\begin{aligned}
-T \psi\left(q_{\xi_{0}}\right)= & \int_{0}^{T}\left(\frac{\partial A}{\partial q_{1}}\left(t, q_{\xi_{0}}\right) \dot{q}_{\xi_{0}} \cdot \dot{q}_{\xi_{0}}-g^{\prime}\left(q_{1, \xi_{0}}\right)-\frac{\partial V_{1}}{\partial q_{1}}\left(t, q_{\xi_{0}}\right)\right) \\
= & \int_{0}^{T} \sum_{k=1}^{N} \frac{\partial A}{\partial q_{k}}\left(t, q_{\xi_{0}}\right) \dot{q}_{\xi_{0}} \cdot \dot{q}_{\xi_{0}}-\int_{0}^{T}\left(g^{\prime}\left(q_{1, \xi_{0}}^{0}+\xi_{0}\right)-g^{\prime}\left(\xi_{0}\right)\right) \\
& -T g_{m}^{\prime}-\int_{0}^{T} \sum_{k=1}^{N} \frac{\partial V_{1}}{\partial q_{k}}\left(t, q_{\xi_{0}}\right) \\
\geq & -T g_{m}^{\prime}-\left[T V_{M}+\lambda_{1} \int_{0}^{T}\left|\dot{q}_{\xi_{0}}\right|^{2}+\frac{l T^{3 / 2}}{2 \pi}\left\|\dot{q}_{1, \xi_{0}}\right\|_{2}\right] .
\end{aligned}
$$


So, by the estimate of Lemma 1.3 we conclude

$$
\begin{aligned}
-T \psi\left(q_{\xi_{0}}\right) & \geq-T\left[g_{m}^{\prime}+V_{m}+\frac{\lambda_{1} T}{\left(\lambda_{0} \pi\right)^{2}}\left(\left\|f_{0}\right\|_{2}+\sqrt{T} L\right)^{2}+\frac{l T^{3 / 2}}{2 \lambda_{0} \pi^{2}}\left(\left\|f_{0}\right\|_{2}+\sqrt{T} L\right)\right] \\
& \geq-T\left[g_{m}^{\prime}+V_{M}+\frac{T}{\lambda_{0} \pi^{2}}\left(\left\|f_{0}\right\|_{2}+\sqrt{T} L\right)\left(\frac{\lambda_{1}}{\lambda_{0}}\left(\left\|f_{0}\right\|_{2}+\sqrt{T} L\right)+\frac{l}{2} \sqrt{T}\right)\right] \\
& >0 .
\end{aligned}
$$

That is, $d \leq \psi\left(q_{\xi_{0}}\right) \leq g_{m}^{\prime}+V_{M}+\mu_{T}<0$. Similarly, if we let $\xi_{1} \in\left[0, T_{1}\right]$ such that $g^{\prime}\left(\xi_{1}\right)=g_{M}^{\prime}$, we have

$$
\begin{aligned}
T \psi\left(q_{\xi_{1}}\right)= & -\int_{0}^{T} \sum_{k=1}^{N} \frac{\partial A}{\partial q_{k}}\left(t, q_{\xi_{1}}\right) \dot{q}_{\xi_{1}} \cdot \dot{q}_{\xi_{1}}+\int_{0}^{T}\left(g^{\prime}\left(q_{1, \xi_{1}}^{0}+\xi_{1}\right)=g^{\prime}\left(\xi_{1}\right)\right) \\
& +g_{M}^{\prime} T+\int_{0}^{T} \sum_{k=1}^{N} \frac{\partial V_{1}}{\partial q_{k}}\left(t, q_{\xi_{1}}\right) \\
\geq & g_{M}^{\prime} T+V_{m} T-\frac{T^{2}}{\lambda_{0} \pi^{2}}\left(\left\|f_{0}\right\|_{2}+\sqrt{T} L\right)\left(\frac{\lambda_{1}}{\lambda_{0}}\left(\left\|f_{0}\right\|_{2}+\sqrt{T} L\right)+\sqrt{T} \frac{l}{2}\right)>0 .
\end{aligned}
$$

This yields $D \geq g_{M}^{\prime}+V_{m}-\mu_{T}>0$. The conclusion now easily follows from Theorem 1.

\section{ApPlication to the $N$-PENDULUM EQUATION}

Given $m_{i}>0, l_{i}>0, i=1, \ldots, N$, set $M_{i}=\sum_{j=i}^{N} m_{j}$. Theorem 2 applies to the Lagrangian

$$
\mathscr{L}(q, \xi)=\frac{1}{2} A(q) \xi \cdot \xi+g \sum_{k=1}^{N} l_{k} M_{k} \cos q_{k}
$$

where $A(q)=\left\{a_{i, j}(q)\right\}_{i, j=1, \ldots, N}$ and

$$
a_{i, j}(q)=a_{i, j}\left(q_{1}, \ldots, q_{N}\right)=M_{\max (i, j)} l_{i} l_{j} \cos \left(q_{i}-q_{j}\right)
$$

which corresponds to the mechanical system of $N$-coplanar penduli with masses $m_{i}$ and length $l_{i}, i=1, \ldots, N$. We obtain

Corollary 1. Let $f_{0}(t)=\left(f_{1}(t), \ldots, f_{N}(t)\right) \in \mathbf{R}^{N}$ as in Theorem 1. Assume

$$
M_{1} l_{1}-\sum_{k=2}^{N} M_{k} l_{k}=\gamma>0
$$

and

$$
\frac{T^{3 / 2} M_{1} l_{1}}{2 \lambda_{0} \pi^{2}}\left(\left\|f_{0}\right\|_{2}+\sqrt{T} M_{1} l_{1}\right)<\gamma
$$

where $\lambda_{0}$ is the elliptic constant of the matrix $A=A(q)$ given in (1.8). There exist constants $d<0<D$ (depending on $m_{i}, l_{i}, f_{i}, i=1, \ldots, N$ and $T$ ) such 
that problem $(1)_{c}$ with $\mathscr{L}$ given in (1.7) admits at least two distinct solutions if $c \in(d, D)$ and at least one solution if $c=d$ or $c=D$. Furthermore,

$$
\begin{aligned}
d & \leq-\gamma+\frac{M_{1} l_{1} T^{3 / 2}}{2 \lambda_{0} \pi^{2}}\left(\left\|f_{0}\right\|_{2}+\sqrt{T} M_{1} l_{1}\right)<0 \\
& <\gamma-\frac{M_{1} l_{1} T^{3 / 2}}{2 \lambda_{0} \pi^{2}}\left(\left\|f_{0}\right\|_{2}+\sqrt{T} M_{1} l_{1}\right) \leq D .
\end{aligned}
$$

Proof. Just notice that in this case $L=l=M_{1} l_{1}$ and $\sum_{k=1}^{N} \frac{\partial A(q)}{\partial q_{k}} \xi \cdot \xi=0$ for all $q, \xi \in \mathbf{R}^{N}$; so we can take $\lambda_{1}=0$.

Finally, concerning the corollary as stated in the Introduction, notice that it is exactly Corollary 1 with $N=2$, where $\lambda_{0}$ has been explicitly computed and given by $(0.4)$.

\section{REFERENCES}

1. A. Ambrosetti and P. Rabinowitz, Dual variational methods in critical point theory, J. Funct. Anal. 14 (1973), 343-387.

2. A. Capozzi, D. Fortunato, and A. Salvatore, Periodic solutions of Lagrangian systems with bounded potential, J. Math. Ann. Appl. 124 (1987), 482-494.

3. K. C. Chang, Y. Long, and E. Zehnder, Forced oscillations for the triple pendulum, Preprint.

4. G. Fournier and M. Willem, Multiple solutions of the forced double pendulum equations, Ann. Inst. H. Poincaré Anal. Non Linéaire 6 (1989), 259-282.

5. D. Guo, J. Sun, and G. Qi, Some extension of the mountain-pass lemma, Differential Integral Equations 1 (1988), 351-358.

6. H. Hofer, A geometric description of the neighborhood of a critical point given by the Mountain-Pass Theorem, J. London Math. Soc. 31 (1985), 566-570.

7. J. Mawhin and M. Willem, Multiple solutions of the periodic boundary value problem for some forced pendulum-type equations, J. Differential Equations 52 (1984), 264-287.

8. R. Palais, The Lusternik-Schnirelman theory on Banach manifolds, Topology 5 (1966), 115132.

9. P. Rabinowitz, On a class of functionals invariant under $a \mathbf{Z}^{n}$ action, Trans. Amer. Math. Soc. 310 (1988), 303-311.

10. G. Tarantello, Multiple forced oscillations for the $N$-pendulum equation, Comm. Math. Phys. 132 (1990), 499-517.

11. G. Tarantello, On the number of solutions for the forced pendulum equation, J. Differential Equations 80 (1989), 79-93.

Department of Mathematics, University of California, Berkeley, California 94720

Current address: Department of Mathematics, Carnegie Mellon University, Pittsburgh, Pennsylvania 15213 